Rev. Inst. Med. Trop. Sao Paulo

\title{
CORRESPONDENCE
}

\section{FALSE-NEGATIVE DENGUE CASES}

September 24, 2014

Dear Editor, we would like to discuss on the recent publication on false-negative dengue cases ${ }^{1}$. ACOSTA et al. ${ }^{1}$ reported that "Health care providers should therefore be aware of samples tested negative by NS1 antigen assays, especially when clinical symptoms and other laboratory data results show evidence of dengue infection". In fact, the problem of false-negative in dengue diagnosis is an important issue for discussion. First, the problem of the NS1 assay in dengue diagnosis is noted worldwide ${ }^{2}$. Combining with other immunological test is suggested for improvement of diagnostic accuracy $^{2}$. Also, it should be noted that the diagnosis of dengue in many tropical countries is usually a presumptive diagnosis without the laboratory confirmation $^{3}$. This implies that there can be both false-positive and false-negative of dengue diagnosis. Sometimes, other arboviral infection such as Chikungunya virus infection can have the similar clinical feature to dengue and mis-diagnosed as dengue. In addition, there can be more than one arboviral infection in a case (such as co-infection between dengue and Mayaro virus infection) ${ }^{4}$. Nevertheless, the definitive diagnosis is more useful for epidemiological purpose than therapeutic purpose. The symptomatic and supportive treatment is usually the basic effective management for the patient with dengue and other similar arboviral infections.

Sim Sai TIN(1) \& Viroj WIWANITKIT(2)

(1) Medical Center, Shantou, China. (2) Visiting professor, Hainan Medical University, China.

Correspondence to: Professor Sim Sai Tin

Medical Center, Shantou, China.

E-mail: simsaitin@gmail.com

\section{REFERENCES}

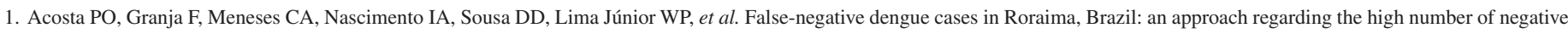
results by NS1 Ag kits. Rev Inst Med Trop Sao Paulo. 2014;56:447-50.

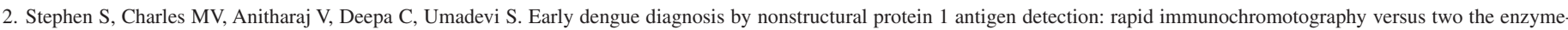
linked immunosorbent assay kits. Indian J Pathol Microbiol. 2014;57:81-4.

3. Wiwanitkit V. Dengue fever: diagnosis and treatment. Expert Rev Anti Infect Ther. 2010;8:841-5.

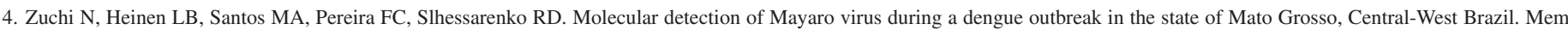
Inst Oswaldo Cruz. 2014;109:820-3. 